\title{
Regulator of G-protein signaling 5 (RGS5) protein: a novel marker of cancer vasculature elicited and sustained by the tumor's proangiogenic microenvironment
}

\author{
Antonietta Silini - Carmen Ghilardi • Sara Figini • Fabio Sangalli • \\ Robert Fruscio - Regine Dahse - R. Barbara Pedley • Raffaella Giavazzi • \\ MariaRosa Bani
}

Received: 28 June 2011/Revised: 10 October 2011/Accepted: 11 October 2011/Published online: 1 December 2011

(C) The Author(s) 2011. This article is published with open access at Springerlink.com

\begin{abstract}
We previously identified regulator of G-protein signaling 5 (RGS5) among several genes expressed by tumor-derived endothelial cells (EC). In this study, we provide the first in vivo/ex vivo evidence of RGS5 protein in the vasculature of ovarian carcinoma clinical specimens and its absence in human ovaries. Consistent with this, we show higher amounts of Rgs5 transcript in EC isolated from human cancers (as opposed to normal tissues) and demonstrate that expression is sustained by a milieu of factors typical of the proangiogenic tumor environment, including vascular endothelial growth factor (VEGF) and basic fibroblast growth factor (FGF-2). Supporting these
\end{abstract}

A. Silini and C. Ghilardi contributed equally to this work.

Electronic supplementary material The online version of this article (doi:10.1007/s00018-011-0862-8) contains supplementary material, which is available to authorized users.

A. Silini · C. Ghilardi · S. Figini · R. Giavazzi $(\bowtie) \cdot$ M. Bani Department of Oncology, Laboratory of Biology and Treatment of Metastases, MarioNegri Institute for Pharmacological Research, via Giuseppe La Masa 19, 20156 Milan, Italy e-mail: raffaella.giavazzi@marionegri.it

F. Sangalli

Department of Biomedical Engineering, Laboratory of Renal Biophysics, MarioNegri Institute for Pharmacological Research, Bergamo, Italy

R. Fruscio

Clinic of Obstetrics and Gynecology, San Gerardo Hospital, University of Milan Bicocca, Monza, Italy

R. Dahse

HELIOS Clinics, Institute of Pathology, Erfurt, Germany

R. B. Pedley

UCL Cancer Institute, University College London, London, UK findings, we show elevated levels of Rgs5 mRNA in the stroma from strongly (as opposed to weakly) angiogenic ovarian carcinoma xenografts and accordingly, we also show more of the protein associated to the abnormal vasculature. RGS5 protein predominantly colocalizes with the endothelium expressing platelet/endothelial cell adhesion molecule-1 (PECAM-1/CD31) and to a much lesser extent with perivascular/mural cells expressing platelet-derived growth factor receptor-beta (PDGFR- $\beta$ ) or alpha smooth muscle actin ( $\alpha \mathrm{SMA})$. To toughen the relevance of the findings, we demonstrate RGS5 in the blood vessels of other cancer models endowed with a proangiogenic environment, such as human melanoma and renal carcinoma xenografts; to the contrary, it was undetectable in the vasculature of normal mouse tissues. RGS5 expression by the cancer vasculature triggered and retained by the proangiogenic microenvironment supports its exploitation as a novel biomarker and opens the path to explore new possibilities of therapeutic intervention aimed at targeting tumor blood vessels.

Keywords Tumor-stromal cell interactions . Tumor microcirculation and microenvironment . New targets - Cancer associated endothelial cells . Angiogenesis and endothelial cell function

\section{Introduction}

Vascularization is required for tumor growth and metastasis, and constitutes a key step in the control of cancer progression [1, 2]. Experimental evidence correlates tumor vascularization with high malignancy, and elevated levels of angiogenic factors - such as vascular endothelial growth factor (VEGF) and fibroblast growth factor (FGF-2) - relate 
to poor prognosis [3]. Tumor-induced vessels are structurally abnormal and differ from normal vasculature. They are heterogeneous in organization, structure, and function, and branch irregularly originating a vasculature unevenly distributed and chaotic [4]. These abnormalities reflect their pathological nature and underline the importance of developing novel therapeutic strategies directed against the vascular elements of the tumor stroma in order to selectively target tumor vasculature and inhibit tumor growth [5].

To this end, in a previous investigation aimed at comparing the gene expression profiles of endothelial cells (EC) from tumor and normal human tissues, we demonstrated distinct transcriptional features characterizing the ECs [6]. Regulator of G-protein signaling (RGS) 5 was listed among the transcripts expressed by the EC coming from the tumor environment.

RGS5 is a member of the RGS family of multifunctional GTPase-activating proteins (GAPs) known to negatively regulate the signaling of G-protein coupled receptors (GPCR); it is currently unknown which pathways are regulated by RGS5. In RGS5-transfected smooth muscle cells and fibroblasts, the capability of RGS5 to attenuate the signaling triggered by proteins mediating vascular functions-such as angiotensin II (AngII), endothelin-1 (ET-1), and sphingosine-1-phosphate (S1P) [7-9]—is suggestive of a possible involvement of RGS5 in blood vessel maturation and homeostasis. Rgs5 mRNA expression was shown to be dynamically regulated during the healing of cutaneous wound and hormone-induced ovulation of mice [10]. In mouse models of pancreatic islet carcinogenesis Rgs5 transcript was found in the blood vessels of the late-stage insulinomas but not of the normal or hyperplastic pancreatic islet $[10,11]$, and its deletion (RIP1-Tag5 crossed to RGS5-deficient mice) was shown to normalize the otherwise chaotic and disorganized tumor vasculature [12].

All these observations strongly point to the importance of RGS5 in angiogenesis and for the tumor blood vessels alterations; despite so, very little is known about its pathophysiological involvement in human cancer.

In light of this consideration, we investigated the contribution of RGS5 to tumor angiogenesis and vasculature using ovarian carcinoma as a paradigm. Most importantly, given that in the current literature the information relies solely on gene expression, we evaluated the expression/ localization of the encoded protein.

In this study, we show for the first time the RGS5 protein as a marker of cancer vasculature, in both human clinical specimens and tumor xenografts. We show the involvement of RGS5 in the vascular remodeling occurring within the tumor's angiogenic microenvironment, and its predominant colocalization with the endothelium lining the tumor blood vessels.
Our findings underline the importance of RGS5 in human cancer and thus are of biomedical relevance. The exploration of whether RGS5 may serve as a biomarker or as a therapeutic target of cancer vasculature will provide an exciting avenue of research.

\section{Materials and methods}

In vitro studies

Cells

Human EC from carcinomas (ovarian and renal) and nonneoplastic tissue specimens (adrenal glands and skin) were isolated and cultured as described [6]. The expression of platelet endothelial cell adhesion molecule-1 (PECAM-1/ CD31) and von Willebrand factor (vWF), the ability to uptake low-density lipoproteins (LDL) and to form cordlike structures were assayed in order to verify the endothelial origin of the cells (as described in [6]). Smooth muscle cells from umbilical artery (uaSMC) were purchased from Clonetics (Clonetics-BioWhittaker) and cultured in SmBM Growth Medium following the manufacturer's protocol. HuFb and Malme-3 human fibroblasts were purchased from ATCC and grown in RPMI-1640 medium plus $10 \%$ FBS. All cells were assayed in the presence of $10 \mathrm{ng} / \mathrm{ml}$ of VEGF and EGF, and $2 \mathrm{ng} / \mathrm{ml}$ of FGF-2; unless otherwise specified. RGS5 was assessed as described below.

In vivo studies

Mice

Female NCr-nu/nu mice, 6-8 weeks old, were obtained from Harlan. The mice were maintained under specific pathogen-free conditions, housed in isolated ventilated cages, and handled using aseptic procedures. Procedures involving animals and their care were conducted in conformity with institutional guidelines, in compliance with national (Legislative Decree 116 January 27, 1992, Authorization n.169/94-A issued December 19, 1994, by Ministry of Health), and international laws and policies (EEC Council Directive 86/609, OJ L 358.1, December 12, 1987; Standards for the Care and Use of Laboratory Animals, United States National Research Council, Statement of Compliance A5023-01, 1996).

\section{Tumor xenografts}

Human xenografts of 1A9-VS1 and 1A9-VAS3 ovarian carcinoma, RXF393 renal carcinoma, and WM1552/5p 
melanoma were obtained by subcutaneous injection of $10 \times 10^{6}$ cells in the flanks of NCr-nu/nu mice. 1A9-VS1 (high VEGF) and 1A9-VAS3 (low VEGF) cells were previously originated [13]. RXF393 cells [14] were kindly provided by H. Fiebig (Freiburg, Germany). WM1552/5p cells were selected by A. Silini upon serial transplantations of subcutaneously grown tumors from WM1552C melanoma cells, kindly provided by M. Herlyn (Philadelphia, PA, USA) [15].

\section{Microdissection of stroma from 1A9-VS1 and 1A9-VAS3 ovarian carcinoma xenograft}

Hematoxylin-stained sections $(10 \mu \mathrm{m})$ from frozen tumor tissue were dissected with the PALM Microlaser System (Carl Zeiss); stroma was separated from the parenchyma without selecting any particular type of component (ECs, fibroblasts, etc.) and immediately lysed. RNA extraction and real-time PCR were performed as described below.

Gene expression studies

\section{RNA and real-time PCR}

Total RNA was isolated with RNeasy ${ }^{\circledR}$ Micro Kit (Qiagen) and converted to cDNA with random hexamers (Archive Kit, Applied Biosystems). Gene expression was analyzed by real-time PCR using TaqMan ${ }^{\circledR}$ Gene Expression Assay (Applied Biosystems). Statistical analysis by unpaired twotailed $t$ test and non-parametric Mann-Whitney test was performed on the deltaCt $(\mathrm{dCt})$ values to compare the expression of RGS5 in EC isolated from tumor specimens versus normal tissues, and in microdissected stroma from 1A9-VS1 versus 1A9-VAS3 xenografts. Relative expression was referred to the sample with the highest $\mathrm{dCt}$ and determined by the formula: $2^{-\mathrm{ddCt}}[16]$.

Immunofluorescence studies

\section{Cells}

EC were grown onto coverslips, fixed (4\% paraformaldehyde), permeabilized ( $0.1 \%$ Triton X-100), and stained for RGS5 as specified below. Coverslips were mounted onto glass slides after DAPI $(0.6 \mu \mathrm{g} / \mathrm{ml})$ counterstain.

\section{Tumor xenografts}

Cryostat sections $(8 \mu \mathrm{m})$ were thawed, fixed (acetone), blocked (3\% rabbit serum), and stained for $1 \mathrm{~h}$ with goatanti-human RGS5 (1:250, Santa Cruz Biotechnology). Rinsed sections were incubated with Alexa Fluor 488 rabbit-anti-goat (1:800, Molecular Probes). Additionally, sections were double/triple stained for RGS5 together with rat-anti-mouse $\mathrm{CD} 31$ (1:50, $\mathrm{BD}$ Pharmingen, cloneMEC13.3) or rat-anti-mouse CD140b/PDGFR- $\beta$ (1:500, eBioscience, cloneAPB5) or Cy3-conjugated anti-mouse aSMA (1:200, Sigma), and with secondary antibodies Alexa Fluor 350 goat-anti-rat (1:200, Molecular Probes) or TRITC rabbit-anti-rat (1:200, Sigma) as appropriate.

\section{Clinical specimens}

Tissue sections were handled as described for the tumor xenografts with the exception of assaying serial sections by single color imaging to investigate RGS5, $\alpha$ SMA, and CD31 (Alexa Fluor 488-conjugated mouse-anti-human CD31; 1:100, BD Pharmingen, cloneM89D3). Tissue sections were from patients who underwent surgery at the San Gerardo Hospital, Monza (Italy). Ovarian carcinoma specimens were collected at time of the first debulking surgery from three patients [FIGO stage I and II $(n=2)$ ] that did not receive neoadjuvant chemotherapy, and from one patient relapsing after surgery and chemotherapy. Nonneoplastic ovaries $(n=3)$ were from postmenopausal patients who underwent hysterectomy and bilateral salpingo-oophorectomy for genital prolapse. Tissues were immediately flash-frozen. The research was approved by the relevant ethics committees and all human participants gave written informed consent.

\section{Fluorescence imaging: quantitative assessment of RGS5 protein expression}

RGS5 fluorescence signal was expressed as "area density" as described [17] Briefly, tissue sections stained for RGS5 (or only with the secondary antibody) were viewed with a $10 \times$ objective lens magnification by the BX61 microscope (Olympus), and digital images captured and converted into 8-bit grayscale images using ImageJ software http://rsbweb.nih.gov/ij/. The "area density" was calculated by dividing the number of RGS5 positively stained pixels (i.e., with brightness greater than an arbitrary threshold) by the number of pixels comprising the entire sectional area. The threshold distinguishing the "RGS5positive signal" was the brightness value below which $99 \%$ of pixels fell in the negative control.

Four human ovarian carcinoma specimens versus three non-neoplastic ovaries, and three 1A9-VS1 versus three 1A9-VAS3 tumors were examined for RGS5 expression. At least three images were quantified for each sample ( $n \geq 9$ sectional areas) and differences in the "area density" between the two groups were evaluated by unpaired two-tailed $t$ test and non-parametric Mann-Whitney test; $p$ values $<0.05$ were considered statistically significant. 
Multiple color fluorescence imaging: overlap of fluorophores

Double- and triple-stained sections of tumor xenografts were examined with the Olympus BX61 epifluorescence microscope, using the appropriate filter-set for the different fluorochrome characteristics. Digital images from the different excitation wavelengths were captured with ColorView II Soft Imaging System digital color camera (Olympus) then co-registered using Adobe Photoshop software (Adobe Systems) to combine multiple colors into a single final image (merge) showing overlapping, fluorescently labeled structures (RGS5, CD31, $\alpha$ SMA, PDGFR- $\beta$ ).

\section{Laser scanning confocal microscopy: colocalization of fluorophores}

Selected multiple-labeled specimens were examined by confocal microscopy to reveal colocalization. Sections were scanned with blue diode, argon-ion, and green helium-neon lasers (405-, 488-, and 543-nm spectral lines, respectively) by sequential scanning and detection mode to eliminate possible bleed-through effect. The images were acquired with a $63 \times$ objective lens magnification by the Zeiss LSM 510 Meta confocal microscope (Carl Zeiss).

\section{Results}

RGS5 expression in human tumor-derived endothelial cells and its modulation by proangiogenic factors

Our previous microarray analysis investigation-aimed at finding novel markers of tumor vasculature-listed RGS5 among the genes highly expressed by tumor-derived EC [6]. In the present study, primary cultures of endothelial cells were investigated by real-time PCR. We demonstrate a statistically significant higher level of Rgs5 transcript in EC isolated from human carcinomas (mostly ovarian) in comparison to EC isolated from non-neoplastic tissue (adrenal glands and skin). As shown in Fig. 1a, the relative expression of Rgs5 mRNA is at least ten times higher in respect to normal tissue-derived $\mathrm{EC}$, and also to commercially available human smooth muscle cells and fibroblasts (that performed as the normal tissue-derived EC).

To mimic the milieu in which the EC are embedded within a solid tumor's microenvironment, stromal cells were cultured with factors acknowledged to be typical of the cancer/angiogenic environment, such as VEGF, FGF-2, and EGF. We demonstrate that the removal of such an environment from the tumor-derived EC results in a robust decrease of Rgs5 mRNA expression; as shown in Fig. 1b, a diminished amount of RGS5 transcript is consistently measured in all the EC evaluated.

The primary cultures of EC express the protein encoded by RGS5 (Fig. 1c), in addition to forming cord/tubular structures, uptaking LDL, expressing vWF and PECAM-1/ CD31, typical features of endothelial cells.

These results demonstrate the expression of RGS5 by EC isolated from human cancer specimens that is dynamically modulated by the surroundings, and in particular elicited by a milieu of proangiogenic factors, suggesting that in an in vivo setting its expression by the cancer vasculature is sustained by the microenvironment.

RGS5 protein expression in the cancer vasculature of human clinical specimens

Sections from ovarian carcinomas and non-neoplastic ovaries (patients' biopsies) were investigated to assess RGS5 protein by immunofluorescence. All the cancer specimens stain positive for RGS5 (Fig. 2a, b), whereas no clearly visible signal is detectable in any of the ovaries (Fig. 2a). Imaging analyses reveal that RGS5 fluorescence covers $7.3 \%$ of the area in ovarian carcinoma specimens and less than $1 \%$ in healthy ovaries (Fig. 2b).

RGS5 staining clearly excludes the parenchyma (Fig. 2a); the pattern of expression being reminiscent of vessel-like structures (Fig. 2b). Accordingly, serial sections of the same specimens indicate that RGS5 positivity resembled that of CD31 and $\alpha$ SMA (Fig. 2c), markers of vascular endothelium and perivascular mural cells.

These results show the expression of RGS5 protein by the vasculature of ovarian carcinoma clinical specimens and not by the ovaries, which is suggestive of RGS5 as a marker of cancer vasculature, which expression is triggered/sustained by the tumor microenvironment.

RGS5 mRNA and protein expression in the vasculature of strongly versus weakly angiogenic tumor xenografts

RGS5 was investigated in an experimental in vivo setting where the same tumor type is endowed with either high or low angiogenic potential: human ovarian carcinoma cells moderately expressing VEGF, FGF-2, and PIGF (1A9VAS3) and their isogenic variant (1A9-VS1) transfected to over-express VEGF, such that high amounts are measured in the plasma of tumor-bearing mice [13], as occurs in ovarian cancer patients [18]. The vasculature of 1A9-VS1 xenografts abounds of abnormally dilated blood vessels and is quite heterogeneous, as opposed to the weakly angiogenic 1A9-VAS3 tumors comprising the same number of blood vessels but displaying a rather homogeneous, less abnormal vascular network [13]. 


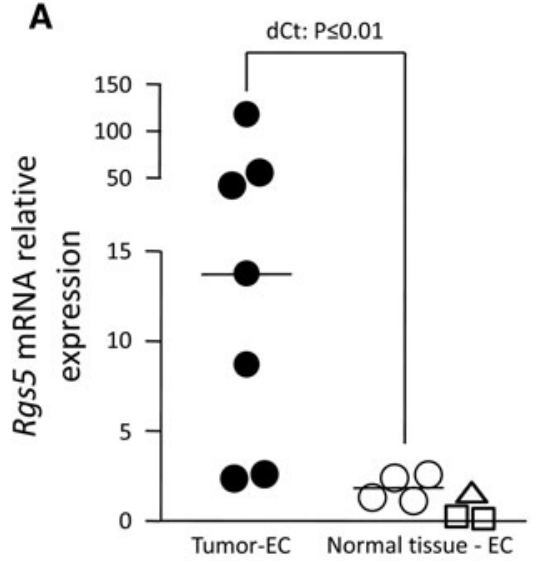

C RGS5
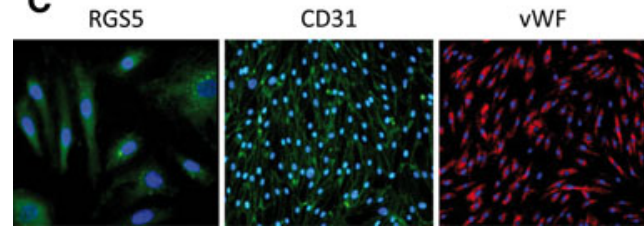

Fig. 1 RGS5 expression by human endothelial cells (EC). a Rgs5 mRNA is much more expressed in tumor-than in normal tissuederived EC. EC-isolated from human carcinomas or normal tissue specimens-were grown in presence of the proangiogenic factors VEGF, FGF-2 and EGF and investigated to assess Rgs5 mRNA by real-time PCR. Statistical analysis evaluated the $\mathrm{dCt}$ values. The $y$ axis indicates the relative expression: tumor-EC $=$ black circles, normal tissue-EC $=$ white circles, lines $=$ median values . Fibroblasts (white squares) and uaSMC (white triangle) performed as the normal tissue EC. b Rgs5 mRNA expression by tumor-derived EC is fueled

Rgs5 mRNA is expressed by the stroma microdissected from both tumor variants; most importantly the amount of transcript is statistically different (mean dCt 8.6 vs. 12) and much higher in the stroma of 1A9-VS1 (Fig. 3a).

The expression of RGS5 protein is marked in both xenografts and clearly excludes the parenchyma (Fig. 3b) as already shown for the clinical specimens (Fig. 2). The staining pattern of RGS5 is evocative of the different vasculature, one being heterogeneous in distribution and diameters (1A9-VS1) and the other having a more homogeneous network (1A9-VAS3). Protein was quantified and is twofold higher in the VEGF-rich tumor environment; RGS5 fluorescence covers $4.6 \%$ of the sectional area in 1A9-VS1 and only $2.4 \%$ in 1A9-VAS3 (Fig. 3b), the difference being statistically significant.

Altogether, these results show the RGS5 relationship with the abnormal vasculature of the 1A9-VS1 tumor xenografts triggered/sustained by the microenvironment's proangiogenic rich milieu and are suggestive of the RGS5
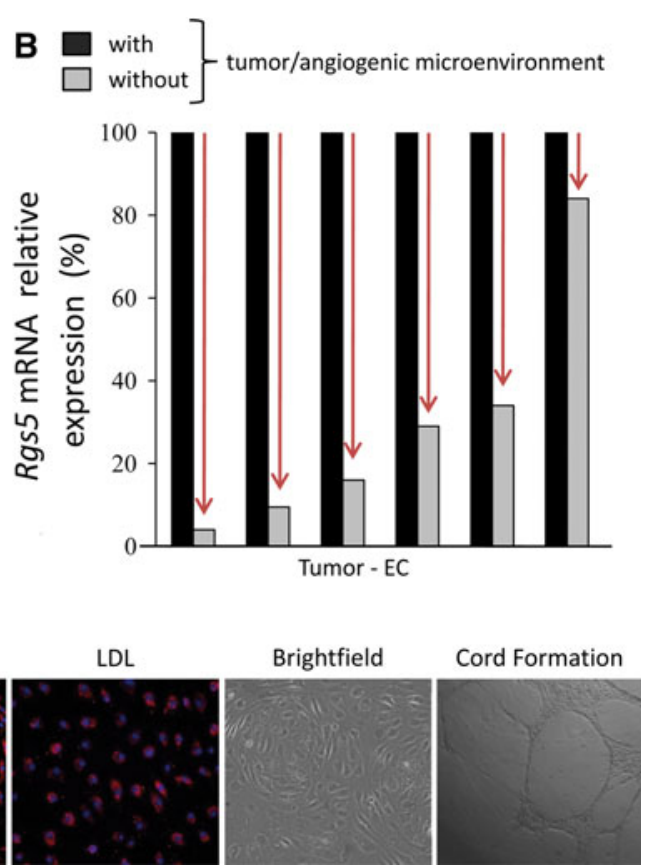

by angiogenic growth factors. Rgs5 mRNA was evaluated in tumorderived EC grown in presence of a tumor/angiogenic microenvironment (as in a) and subsequent to its withdrawal. The arrows indicate the declining of expression. $\mathbf{c}$ RGS5 protein is expressed by tumor-EC growing in vitro. RGS5 protein (green, first panel) in representative EC isolated from an ovarian carcinoma specimen whose endothelial origin was verified by positive staining for CD31 (green) and vWF (red), by LDL uptake (red), by morphology (brightfield) and cord formation

functional implication in the abnormal remodeling of blood vessel.

RGS5 protein expression by the endothelium of ovarian carcinoma xenografts

RGS5 protein was investigated together with the EC marker CD31, to provide an in vivo understanding of the vessel-associated cell source. The staining pattern of RGS5 and CD31 is pretty much identical (Fig. $4 \mathrm{a}, \mathrm{b}$ ), consistent with the overlapping of colors in the merge image (Fig. 4c). Their co-expression was confirmed by confocal microscopy. Colocalization of RGS5 with CD31 is revealed in double-stained sections (Fig. 4f) as well as in triple-stained sections (Fig. 4i), where colocalization of RGS5 with CD31 is shown in areas rich in both CD31- and $\alpha$ SMA-positive vascular structures.

These results demonstrate that the endothelium lining the blood vessels of human ovarian cancer xenografts 
Fig. 2 RGS5 protein

expression by the vasculature of human clinical specimens. In human clinical specimens, RGS5 protein is expressed in ovarian carcinomas but absent in non-neoplastic ovaries.

a Representative images (scale bars $100 \mu \mathrm{m}$ ) illustrating tissue sections immunostained for RGS5 (green) and

counterstained with DAPI (blue) from human ovarian cancer specimens and nonneoplastic ovaries. [Note that the latter is negative for RGS5, but positive for CD31 and aSMA]. Demonstrative H\&E images are shown.

b Quantification of protein ${ }^{*} p<0.05$. Representative images (scale bars $20 \mu \mathrm{m}$ ) illustrating the positive RGS5 staining of clinical specimens. c RGS5 protein

immunofluorescence resembles vessel-like structures.

Representative images (scale bars $100 \mu \mathrm{m}$ ) of serial sections (top vs. bottom) from ovarian carcinoma specimens stained for RGS5, CD31, $\alpha$ SMA

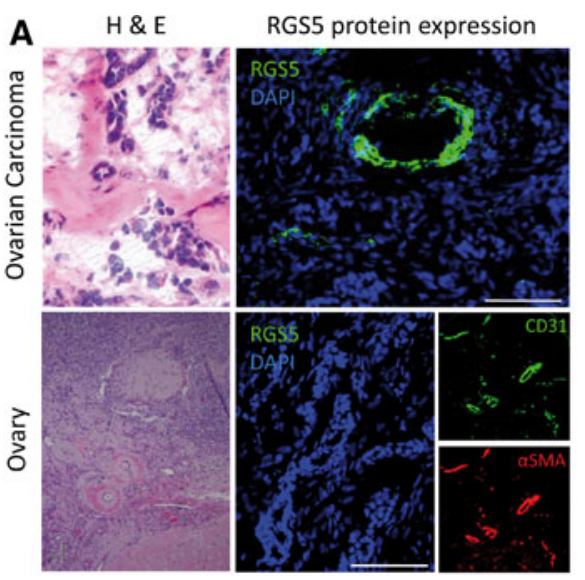

B
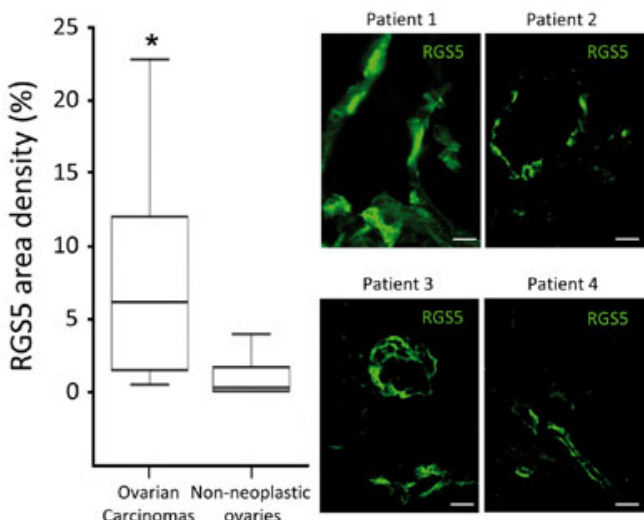

Patient 3
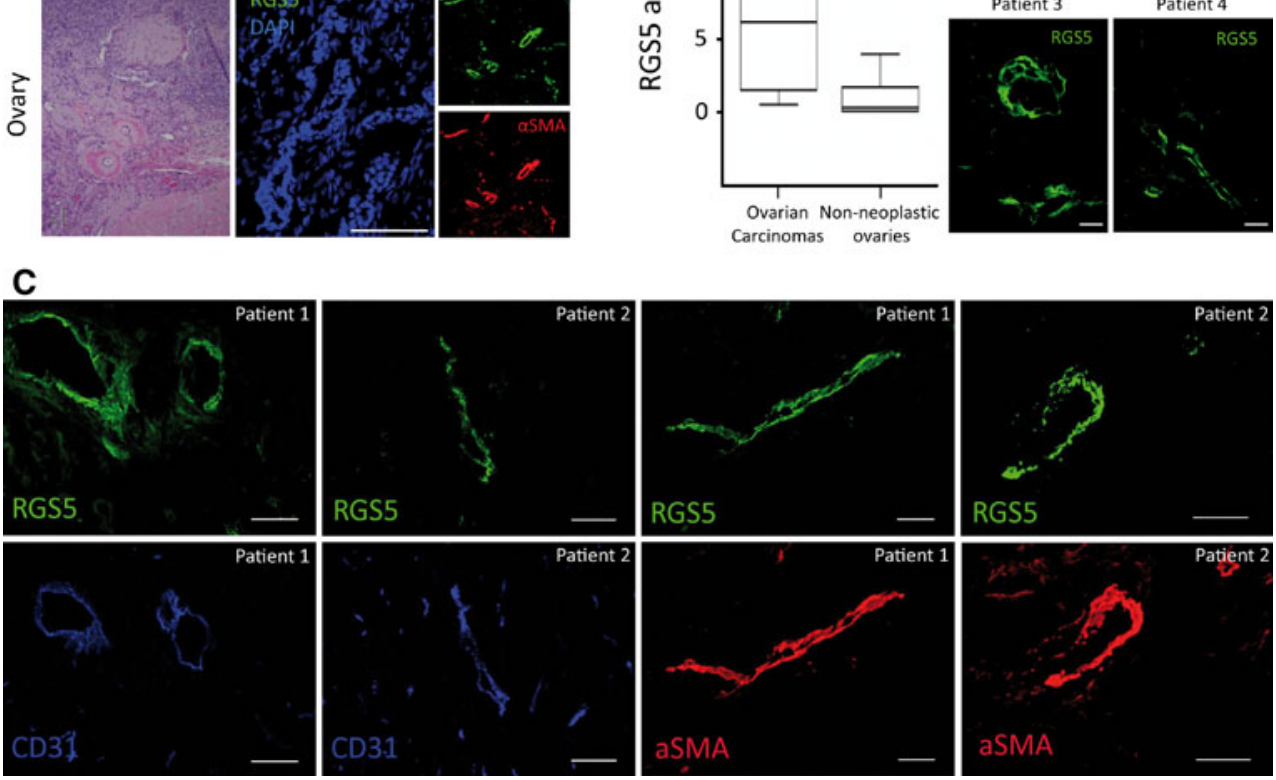

A

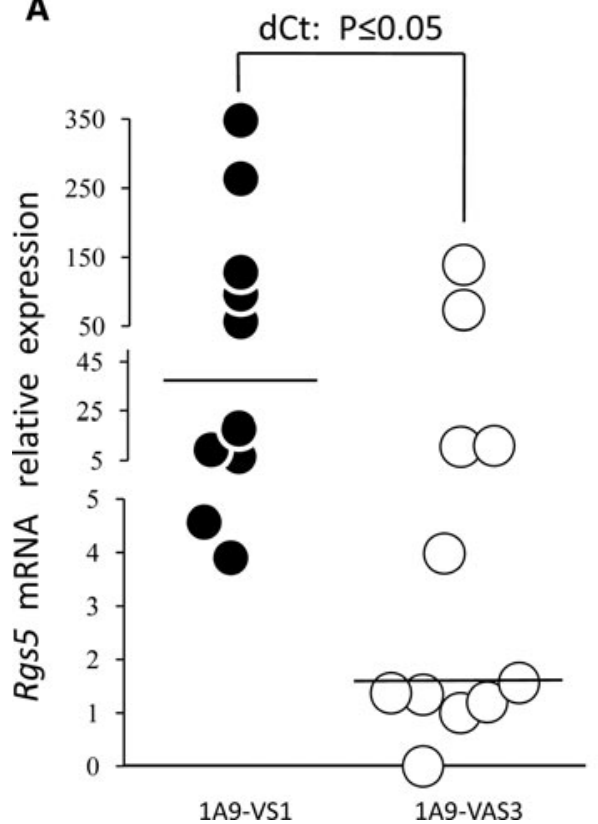

Fig. 3 RGS5 mRNA and protein expression by human ovarian carcinoma xenografts: VEGF-rich compared to VEGF-poor tumors. a Rgs5 mRNA is more expressed in the stroma of 1A9-VS1 than 1A9VAS3 tumors. Stroma was microdissected from 1A9-VS1 and 1A9VAS3 xenografts ( $n=10$ and $n=11$ tumors, respectively) and Rgs5 expression assessed by real-time PCR. Statistical analysis evaluated the dCt values: unpaired two-tailed $t$ test $p=0.017$, non-parametric
B

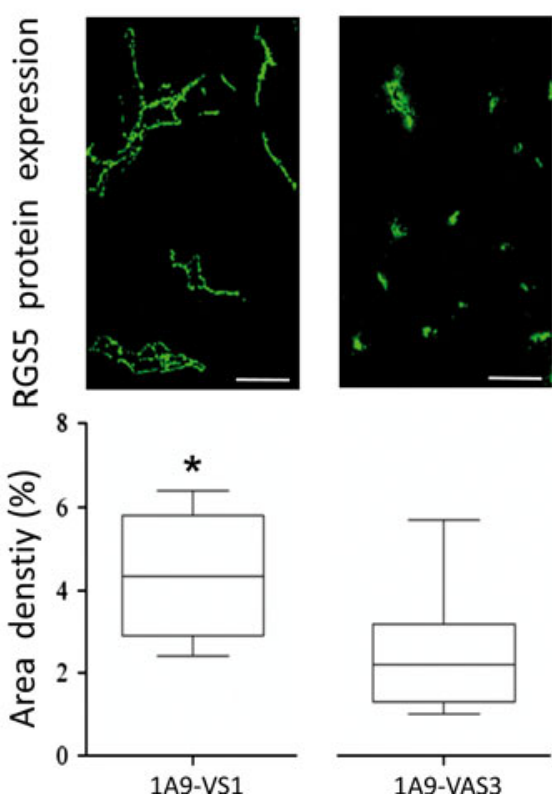

Mann-Whitney test $p=0.02$. The $y$-axis indicates the relative expression referred to the stroma sample with the lowest but detectable expression. b RGS5 protein is more expressed in 1A9VS1 than 1A9-VAS3 tumors. Representative images (scale bars $200 \mu \mathrm{m}$ ) illustrating tissue sections immunostained for RGS5 (green). Quantification of protein:* $p<0.05$. For each tumor variant, at least nine section-images were examined 

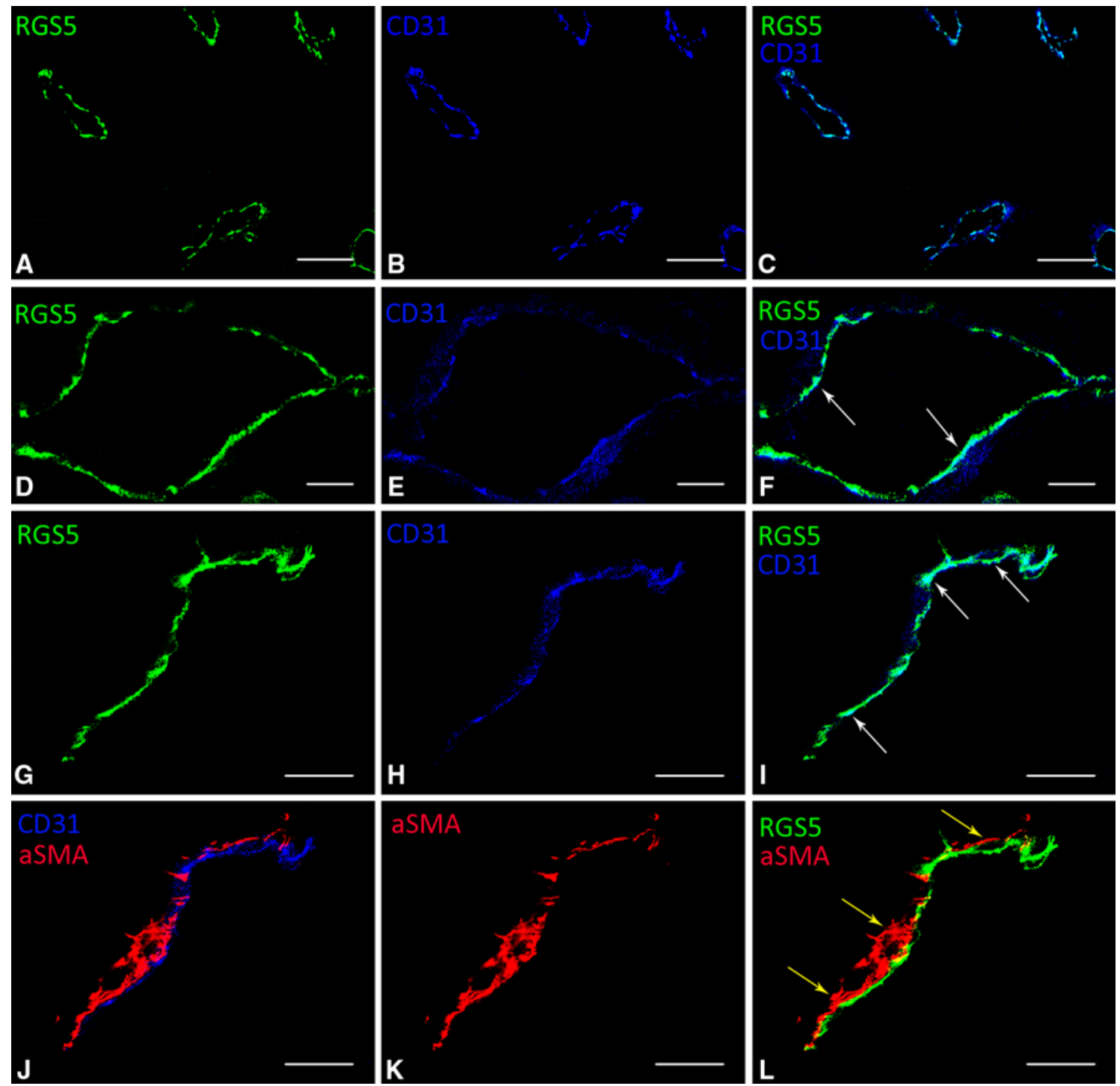

Fig. 4 RGS5 protein is expressed by vascular endothelium of human ovarian carcinoma xenografts. Representative images illustrating 1A9-VS1 tissue sections co-stained with RGS5 (green), CD31 (blue) and/or $\alpha$ SMA (red). a-c Epifluorescence microscopy (scale bars $100 \mu \mathrm{m}$ ); the merge image (c) shows RGS5-CD31 overlap (light

expresses RGS5, congruent with the ovarian carcinoma clinical specimens and as revealed by the in vitro results.

RGS5 protein expression by the vascular endothelium of different tumor types

Having demonstrated that RGS5 is expressed by the EC of abnormal tumor vasculature in ovarian cancer fueled by the angiogenic microenvironment, we assessed whether the same could be observed in other angiogenic tumor models, namely human renal carcinoma RXF393 and melanoma WM1552/5p xenografts. These tumors inherently produce human VEGF (measurable in the plasma of tumor-bearing mice) and moderately express also FGF-2 or PDGF-B. blue). d-l Confocal microscopy (scale bars $20 \mu \mathrm{m})$ of double- $(\mathbf{d}-$ f) and triple- (g-l) stained sections. Images show colocalization of RGS5 with CD31 (f and i, white arrows) mostly excluding $\alpha$ SMA (l, yellow arrows)

We demonstrate the expression of RGS5 protein and its association with the endothelium in both these xenografts. The vascular network is different between the two tumors (Fig. 5b, e), and RGS5 recapitulate the same staining patterns (Fig. 5a, d); indeed the merge images show a great degree of RGS5-CD31 overlap in both RXF393 and WM1552/5p (Fig. 5c, f). Moreover, triple-stained sections confirm that RGS5 overlaps with CD31 (Fig. 5i) rather than with $\alpha$ SMA (Fig. 5l). Altogether, these results provide strong evidence that the endothelium lining the blood vessels of cancer expresses RGS5. Conversely, no clear signal of RGS5 staining in association with the vasculature of normal mouse organs is visible (supplementary material 1). 

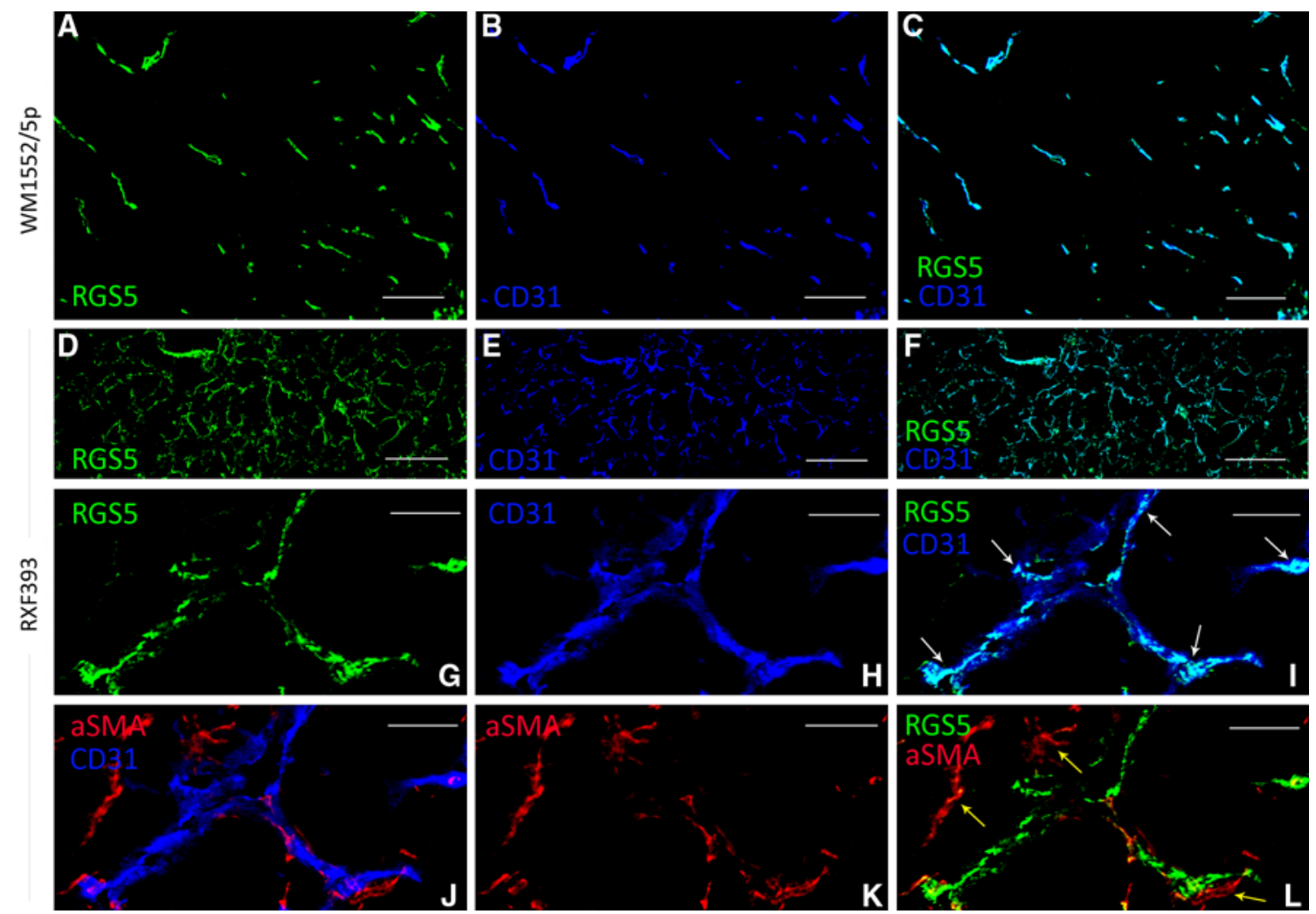

Fig. 5 RGS5 protein is expressed by vascular endothelium of human melanoma and renal carcinoma xenografts. Representative images illustrating WM1552/5p and RXF393 tissue sections co-stained with RGS5 (green), CD31 (blue) and/or $\alpha \mathrm{SMA}$ (red). a-f (scale bars

RGS5 protein can colocalize with perivascular cells comprising the tumor blood vessels, too

Given that the expression of Rgs5 mRNA had been associated with mural cells such as pericytes/vSMC in tumors of RIP1-Tag2 mice [11], we double stained the human xenografts either with RGS5 and $\alpha$ SMA or with RGS5 and PDGFR- $\beta$. Representative merge images show that RGS5 protein overlaps to a certain extent with $\alpha \mathrm{SMA}$ and with PDGFR- $\beta$ positive staining (Fig. 6c, i). The expression of RGS5 by PDGFR- $\beta$ and $\alpha$ SMA-positive cells was confirmed by confocal microscopy. As shown in Fig. 6, RGS5 staining can colocalize with PDGFR- $\beta$ (Fig. 61) and also with $\alpha$ SMA (Fig. 6f), signifying that the mural cells associated with the abnormal tumor vasculature are capable of expressing RGS5.

\section{Discussion}

This study demonstrates RGS5 as a protein characterizing the endothelium of human cancer, and involved in the remodeling of the vasculature occurring during pathological angiogenesis triggered by the tumor microenvironment.
$100 \mu \mathrm{m}$ ), RGS5-CD31 overlap (light blue) is shown for both tumors in the merge images (c and $\mathbf{f})$. $\mathbf{g}-\mathbf{l}$ (scale bars $20 \mu \mathrm{m}$ ), note the great overlapping of RGS5 to CD31 (i, white arrows) mostly excluding the $\alpha$ SMA positive stain (l, yellow arrows) in the triple stained section

Thus, our results open the path to explore new possibilities to target/affect the cancer vasculature for therapeutic intervention and to exploit novel molecules, which could serve as clinical biomarkers.

RGS5 is one of the many modulators of GPCR signaling, but the regulatory functions and the role remain to be elucidated. Recently, in RIP1-Tag mice Rgs5 mRNA was shown associated to the vascular bed of late-stage insulinomas but not of the normal/hyperplastic pancreatic islets [10, 11]; its ablation (RIP1-Tag5 crossed to RGS5 knockout mice) was shown to "normalize" the otherwise abnormal, chaotic, and disorganized vasculature of insulinomas [12]. These observations altogether suggest a key role of RGS5 in the blood vessel remodeling occurring during carcinogenesis. Despite this, its connection with human cancer remains largely elusive.

We now show the RGS5 protein in the vasculature of ovarian carcinoma clinical specimens and its absence in non-neoplastic ovaries, suggestive of its involvement in the malignant progression of human cancer. By focusing on the protein, our results strengthen the currently published observations pertaining to clinical specimens, which are limited to in situ hybridization and refer to different cancer types; Rgs5 mRNA was detected in sinusoidal endothelium 

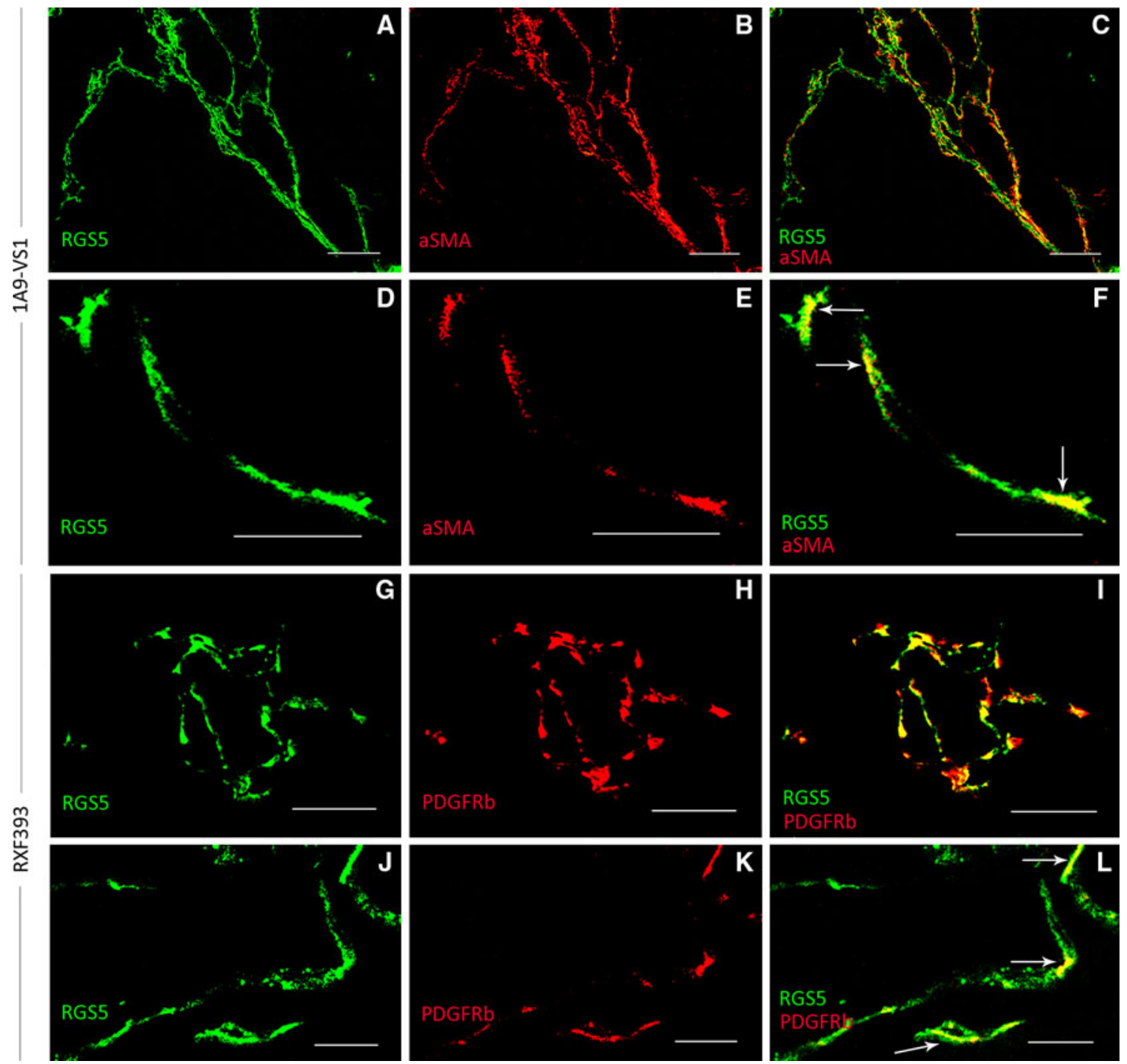

Fig. 6 RGS5 protein is expressed by vascular mural cells. Representative images illustrating 1A9-VS1 and RXF393 tissue sections co-stained with RGS5 (green), and either $\alpha$ SMA or PDGFR- $\beta$ (red). a-c and $\mathbf{g - i}$ Epifluorescence microscopy (scale bars $100 \mu \mathrm{m}$ ); the

of hepatocellular carcinoma but not in normal and cirrhotic liver [19], and likewise in renal cell carcinoma vasculature as opposed to the adjacent kidney [20]. Consistent with the in vivo results, we prove that human vascular cells growing in vitro express RGS5, but most importantly we demonstrate that the transcript is more highly expressed by endothelial cells isolated from cancer specimens (mainly ovarian but including a renal carcinoma) than from nonneoplastic tissues. This observation confirms our previous gene expression profiling investigation, concerning a different collection of ECs, [6] that listed RGS5 among the genes distinguishing ovarian carcinomas-derived EC, and substantiate another microarray study similarly listing RGS5 as one of the EC genes characterizing hepatocellular merge images show the overlap (yellow) of RGS5- $\alpha$ SMA (c) or RGS5-PDGFR- $\beta$ (i). d-f and $\mathbf{j}-\mathbf{l}$ Confocal microscopy (scale bars $10 \mu \mathrm{m}$ ) show colocalization of RGS5 (white arrows) with $\alpha \mathrm{SMA}$ (f) or with PDGFR- $\beta$ (1)

carcinoma [19]. Altogether, these findings demonstrate RGS5 as a marker of cancer-associated EC, suggesting the possibility that it may serve as clinical biomarker.

All the observations point to a link between RGS5 expression and the environment in which the cancer-associated EC are embedded, which is rich in proangiogenic factors. To toughen this, we demonstrate that removing the in vitro condition mimicking the proangiogenic tumor microenvironment results in the robust decrease of RGS5 expression, thus making the EC isolated from human cancer specimens less "abnormal" and closer to normal EC. Coherent with our findings, in RIP1-Tag5 mice, murine Rgs5 mRNA has been shown to be down-regulated in "less angiogenic" insulinomas, which vasculature resembled that 
of normal pancreatic islet [10]. Consistent with these findings, we also demonstrate a significantly higher transcript expression by the stroma microdissected from ovarian carcinoma tumor xenograft endowed with high, as opposed to low, angiogenic potential. In agreement, we show that the RGS5 protein colocalizes with the endothelium, and most importantly, is more abundant in the VEGF-rich xenografts where the vasculature is irregular and vessels are dilated while it was undetectable in the vasculature of normal mouse organs. Altogether, these findings further consolidate the implication of RGS5 in the abnormal vascular remodeling occurring within the tumor and triggered by the proangiogenic environment. A role perhaps less important in physiological situations such as developmental angiogenesis given that RGS5-deficient mice lack gross histopathological signs of developmental defects and are fertile [12, 21].

It is well known that VEGF is central to the process of angiogenesis [22]. Its expression has been correlated with clinic-pathologic features and patients' survival, and elevated serum/plasma levels of VEGF have been described in cancer patients, including ovarian carcinoma [18, 23]. In the present study, high levels of VEGF circulated in the bloodstream of mice bearing the strong proangiogenic tumor xenografts (melanoma, renal, and ovarian carcinoma), giving additional credit to a translation of our results into a clinical situation.

Whether within the cancer microenvironment as a whole the expression of RGS5 is directly induced by VEGF or, though necessary VEGF may not be on its own sufficient, requiring the intricate surrounding remains to be elucidated. Cancer vasculature is embedded in a milieu of soluble growth factors, and among other things, affected by hypoxia. HUVEC Rgs5 mRNA could not be induced by VEGF alone (data not shown and [24]) but it increased under hypoxia [24]; implying the need for a complex surrounding. Accordingly, in this study, Rgs 5 expression by EC is sustained by the multi-factorial in vitro setting that includes VEGF, FGF-2 and EGF, and likewise-although expressed at high levels and circulating in the bloodstream of tumorbearing mice-VEGF is not the only proangiogenic factor expressed by the tumor cells originating the different xenografts.

VEGF is pivotal in eliciting angiogenesis, but emerging evidence suggests that inhibition of VEGF signaling (the primary target of therapeutics inhibiting angiogenesis) can trigger molecules, such as FGF-2 to initiate a secondary phase of tumor angiogenesis [25]. The identification of novel/alternative ways to target/affect tumor vasculature will therefore be advantageous; hence our findings have significant potential for anticancer therapy as they pose the basis to explore such a possibility.

The localization of RGS5 to the cells constituting the blood vessels is a matter for debate. Rgs5 mRNA had been either associated with $\mathrm{CD} 31 / \mathrm{CD} 34$ positive vasculature in human renal cell carcinoma [20] or established as a marker of pericytes/vSMC rather than of EC in RIP1-Tag mice tumors $[9,10,26]$, predominantly related to immature (PDGFR- $\beta$ positivity) in respect to mature ( $\alpha$ SMA or NG2 positivity) perivascular cells $[11,12]$. All the in vivo studies relied on transcript expression, making the precise cellular localization very difficult given the close association of the different cells composing the blood vessels. Our results provide a conclusive answer: by showing the colocalization of RGS5 protein mainly with CD31 but also with PDGFR- $\beta$ and $\alpha$ SMA of ex vivo specimens from melanoma, renal, and ovarian carcinoma xenografts, we prove that RGS5 can be expressed by both endothelial and pericytes/vSMC cells.

Endothelial and mural cells are implicated in regulating tumor growth, and therefore both suggested as targets in anticancer therapy aimed at disturbing tumor vasculature. Multikinase inhibitors, such as sunitinib and sorafenib, affecting both VEGF receptor-driven EC and PDGF receptor-dependent pericytes, have recently been introduced into clinical practice for the treatment of advanced renal cell carcinoma and hepatocellular carcinoma [27, 28]. RGS5 expression in human clinical specimens, and its specificity to cells comprising the tumor vasculature, makes it a strong candidate for therapeutic intervention. Strategies reverting tumor blood vessel abnormalities (normalization) are regarded as promising anticancer approaches aimed at improving the response to chemotherapy and reducing metastasis [29-31]. It has been shown that ablation of RGS5 from the tumor microenvironment of RIP1-Tag5 mice resulted in tumor blood vessel "normalization" notably improving outcome in response to immunotherapy [12]. Likewise, enhancement of therapeutic effects has been seen when conventional chemotherapy are administered in combination with anti angiogenic or vascular affecting drugs [32-34]. The potential to enhance the efficacy of anti cancer strategies in the absence of RGS5 deserves to be explored.

Acknowledgments The authors wish to thank Giulia Taraboletti for the critical review of the manuscript, and pathologists P. Perego and V. Castiglioni for the H\&E images. This work was supported by the 7th EU Framework Program for Research and Technological Development (FP7) ADAMANT HEALTH-F2-2008-201342, the Italian Association for Cancer Research (AIRC), the CARIPLO Foundation, the Italian Ministry of Health, the Nerina and Mario Mattioli Foundation.

Conflict of interest The authors declare that they have no conflicts of interest.

Open Access This article is distributed under the terms of the Creative Commons Attribution Noncommercial License which permits any noncommercial use, distribution, and reproduction in any medium, provided the original author(s) and source are credited. 


\section{References}

1. Folkman J (1995) Angiogenesis in cancer, vascular, rheumatoid and other disease. Nat Med 1(1):27-31

2. Hanahan D, Weinberg RA (2011) Hallmarks of cancer: the next generation. Cell 144(5):646-674. doi:10.1016/j.cell.2011.02.013

3. Kerbel RS (2005) Therapeutic implications of intrinsic or induced angiogenic growth factor redundancy in tumors revealed. Cancer Cell 8(4):269-271. doi:10.1016/j.ccr.2005.09.016

4. Nagy JA, Chang SH, Dvorak AM, Dvorak HF (2009) Why are tumour blood vessels abnormal and why is it important to know? Br J Cancer 100(6):865-869. doi:10.1038/sj.bjc.6604929

5. Neri D, Bicknell R (2005) Tumour vascular targeting. Nat Rev Cancer 5(6):436-446. doi:10.1038/nrc1627

6. Ghilardi C, Chiorino G, Dossi R, Nagy Z, Giavazzi R, Bani M (2008) Identification of novel vascular markers through gene expression profiling of tumor-derived endothelium. BMC Genomics 9:201. doi:10.1186/1471-2164-9-201

7. Zhou J, Moroi K, Nishiyama M, Usui H, Seki N, Ishida J, Fukamizu A, Kimura S (2001) Characterization of RGS5 in regulation of $\mathrm{G}$ protein-coupled receptor signaling. Life Sci 68(13):1457-1469. doi:S0024320501009390

8. Wang Q, Liu M, Mullah B, Siderovski DP, Neubig RR (2002) Receptor-selective effects of endogenous RGS3 and RGS5 to regulate mitogen-activated protein kinase activation in rat vascular smooth muscle cells. J Biol Chem 277(28):24949-24958. doi:10.1074/jbc.M203802200

9. Cho H, Kozasa T, Bondjers C, Betsholtz C, Kehrl JH (2003) Pericyte-specific expression of Rgs5: implications for PDGF and EDG receptor signaling during vascular maturation. FASEB $\mathbf{J}$ 17(3):440-442. doi:10.1096/fj.02-0340fje

10. Berger M, Bergers G, Arnold B, Hammerling GJ, Ganss R (2005) Regulator of G-protein signaling-5 induction in pericytes coincides with active vessel remodeling during neovascularization. Blood 105(3):1094-1101. doi:10.1182/blood-2004-06-2315

11. Song S, Ewald AJ, Stallcup W, Werb Z, Bergers G (2005) PDGFRbeta+ perivascular progenitor cells in tumours regulate pericyte differentiation and vascular survival. Nat Cell Biol 7(9):870-879. doi:10.1038/ncb1288

12. Hamzah J, Jugold M, Kiessling F, Rigby P, Manzur M, Marti HH, Rabie T, Kaden S, Grone HJ, Hammerling GJ, Arnold B, Ganss R (2008) Vascular normalization in Rgs5-deficient tumours promotes immune destruction. Nature 453(7193):410-414. doi:10.1038/ nature 06868

13. Manenti L, Riccardi E, Marchini S, Naumova E, Floriani I, Garofalo A, Dossi R, Marrazzo E, Ribatti D, Scanziani E, Bani M, Belotti D, Broggini M, Giavazzi R (2005) Circulating plasma vascular endothelial growth factor in mice bearing human ovarian carcinoma xenograft correlates with tumor progression and response to therapy. Mol Cancer Ther 4(5):715-725. doi:10.1158/ 1535-7163.MCT-04-0305

14. Berger DP, Herbstritt L, Dengler WA, Marme D, Mertelsmann R, Fiebig HH (1995) Vascular endothelial growth factor (VEGF) mRNA expression in human tumor models of different histologies. Ann Oncol 6(8):817-825

15. Satyamoorthy K, DeJesus E, Linnenbach AJ, Kraj B, Kornreich DL, Rendle S, Elder DE, Herlyn M (1997) Melanoma cell lines from different stages of progression and their biological and molecular analyses. Melanoma Res 7(Suppl 2):S35-S42

16. Livak KJ, Schmittgen TD (2001) Analysis of relative gene expression data using real-time quantitative PCR and the 2(-Delta Delta C(T)) Method. Methods 25(4):402-408. doi:10.1006/ meth.2001.1262

17. Sennino B, Kuhnert F, Tabruyn SP, Mancuso MR, Hu-Lowe DD, Kuo CJ, McDonald DM (2009) Cellular source and amount of vascular endothelial growth factor and platelet-derived growth factor in tumors determine response to angiogenesis inhibitors. Cancer Res 69(10):4527-4536. doi:10.1158/0008-5472.CAN-083779

18. Manenti L, Paganoni P, Floriani I, Landoni F, Torri V, Buda A, Taraboletti G, Labianca R, Belotti D, Giavazzi R (2003) Expression levels of vascular endothelial growth factor, matrix metalloproteinases 2 and 9 and tissue inhibitor of metalloproteinases 1 and 2 in the plasma of patients with ovarian carcinoma. Eur J Cancer 39(13):1948-1956. doi:S0959804903004271

19. Chen X, Higgins J, Cheung ST, Li R, Mason V, Montgomery K, Fan ST, van de Rijn M, So S (2004) Novel endothelial cell markers in hepatocellular carcinoma. Mod Pathol 17(10):11981210. doi:10.1038/modpathol.3800167

20. Furuya M, Nishiyama M, Kimura S, Suyama T, Naya Y, Ito H, Nikaido T, Ishikura H (2004) Expression of regulator of G protein signalling protein 5 (RGS5) in the tumour vasculature of human renal cell carcinoma. J Pathol 203(1):551-558. doi: 10.1002/path. 1543

21. Nisancioglu MH, Mahoney WM Jr, Kimmel DD, Schwartz SM, Betsholtz C, Genove G (2008) Generation and characterization of rgs5 mutant mice. Mol Cell Biol 28(7):2324-2331. doi:10.1128/ MCB.01252-07

22. Carmeliet P (2005) VEGF as a key mediator of angiogenesis in cancer. Oncology 69(Suppl 3):4-10. doi:10.1159/000088478

23. Chen A, Yang R, Zhang H, Song H (2009) The relationship of EGFR and VEGF mRNA expression in ovarian carcinoma. Clin Oncol Cancer Res 6:100-103

24. Jin Y, An X, Ye Z, Cully B, Wu J, Li J (2009) RGS5, a hypoxiainducible apoptotic stimulator in endothelial cells. J Biol Chem 284(35):23436-23443. doi:10.1074/jbc.M109.032664

25. Casanovas O, Hicklin DJ, Bergers G, Hanahan D (2005) Drug resistance by evasion of antiangiogenic targeting of VEGF signaling in late-stage pancreatic islet tumors. Cancer Cell 8(4):299-309. doi:10.1016/j.ccr.2005.09.005

26. Bondjers C, Kalen M, Hellstrom M, Scheidl SJ, Abramsson A, Renner O, Lindahl P, Cho H, Kehrl J, Betsholtz C (2003) Transcription profiling of platelet-derived growth factor-B-deficient mouse embryos identifies RGS5 as a novel marker for pericytes and vascular smooth muscle cells. Am J Pathol 162(3): $721-729$

27. Escudier B, Eisen T, Stadler WM, Szczylik C, Oudard S, Siebels M, Negrier S, Chevreau C, Solska E, Desai AA, Rolland F, Demkow T, Hutson TE, Gore M, Freeman S, Schwartz B, Shan M, Simantov R, Bukowski RM (2007) Sorafenib in advanced clear-cell renal-cell carcinoma. N Engl J Med 356(2):125-134. doi:10.1056/NEJMoa060655

28. Llovet JM, Ricci S, Mazzaferro V, Hilgard P, Gane E, Blanc JF, de Oliveira AC, Santoro A, Raoul JL, Forner A, Schwartz M, Porta C, Zeuzem S, Bolondi L, Greten TF, Galle PR, Seitz JF, Borbath I, Haussinger D, Giannaris T, Shan M, Moscovici M, Voliotis D, Bruix J (2008) Sorafenib in advanced hepatocellular carcinoma. N Engl J Med 359(4):378-390. doi:10.1056/NEJMoa 0708857

29. Jain RK (2005) Normalization of tumor vasculature: an emerging concept in antiangiogenic therapy. Science 307(5706):58-62. doi: 10.1126/science. 1104819

30. Rolny C, Mazzone M, Tugues S, Laoui D, Johansson I, Coulon C, Squadrito ML, Segura I, Li X, Knevels E, Costa S, Vinckier S, Dresselaer T, Akerud P, De Mol M, Salomaki H, Phillipson M, Wyns S, Larsson E, Buysschaert I, Botling J, Himmelreich U, Van Ginderachter JA, De Palma M, Dewerchin M, ClaessonWelsh L, Carmeliet P (2011) HRG inhibits tumor growth and metastasis by inducing macrophage polarization and vessel normalization through downregulation of PlGF. Cancer Cell 19(1):31-44. doi:10.1016/j.ccr.2010.11.009 
31. Carmeliet P, Jain RK (2011) Principles and mechanisms of vessel normalization for cancer and other angiogenic diseases. Nat Rev Drug Discov 10(6):417-427. doi:10.1038/nrd3455

32. Hurwitz H, Fehrenbacher L, Novotny W, Cartwright T, Hainsworth J, Heim W, Berlin J, Baron A, Griffing S, Holmgren E, Ferrara N, Fyfe G, Rogers B, Ross R, Kabbinavar F (2004) Bevacizumab plus irinotecan, fluorouracil, and leucovorin for metastatic colorectal cancer. N Engl J Med 350(23):2335-2342. doi:10.1056/NEJMoa032691
33. Giavazzi R, Bani MR, Taraboletti G (2007) Tumor-host interaction in the optimization of paclitaxel-based combination therapies with vascular targeting compounds. Cancer Metastasis Rev 26(3-4):481-488. doi:10.1007/s10555-007-9074-y

34. Wagstaff AJ, Keam SJ, McCormack PL (2009) Bevacizumab plus platinum-based chemotherapy: in advanced non-small cell lung cancer. BioDrugs 23(3):187-196. doi:10.2165/00063030200923030-00005 